\title{
The Telenanopresence Manifesto
}

ISSN: 2637-8078

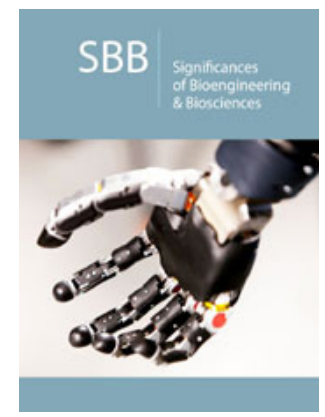

*Corresponding author: Tamvakis I, United Kingdom

Submission: 輻June 14, 2019

Published: 侮June 21, 2019

Volume 3 - Issue 3

How to cite this article: Halidullin O. Climate and Water Cycle in Toilets.Significances Bioeng Biosci.3(3). SBB.000562.2019.

DOI: $10.31031 /$ SBB.2019.03.000562

Copyright@ Halidullin 0, This article is distributed under the terms of the Creative Commons Attribution 4.0 International License, which permits unrestricted use and redistribution provided that the original author and source are credited.

\author{
Angelina NP and Tamvakis I*
}

United Kingdom

\section{Opinion}

Imagine being able to shrink and grow at will and operate as a human being across different scales. Then take this old movie idea and combine it with the emerging technology of telepresence. What you get is the ability of being present, and interact with your surroundings, in a distant location, in a different spatial scale. Realising a telepresence robot in the 1-meter scale is within our grasp, with many such machines being built around the world. What we want to propose here is that Telenanopresence technology, i.e. the ability to make telepresence robots in all different spatial scales, with a focus in the milli-micro scale, will be crucial for the miniaturisation of many industrial processes, and surely a fantastic way for exploring our creativity in the microcosmos. Feynman, in his seminal talk "There's plenty of room at the bottom" [1] exposed the world to the reality of the vast difference in scale between the atoms and us, and how much space for innovation exists in between. He proposed the beautiful idea of recursively exploring this scale-space, by designing and operating a set of tools that is able to make the same set of tools but all somewhat smaller. By this process he wanted to make humanity able, in the end, to manipulate individual atoms, and by combining our fabrication ability across all scales in between, make us master fabricators. It is our contention that his dream was not followed in earnest in the decades since. Marvelous microfabrication techniques like photolithography are changing the world around us but have made shortcuts to the microcosmos that do not allow for the creativity of everyday humans to unfold in each scale in between.

We believe that in order to do that, and do it for everyone on the planet, a standardised human-like human-interfacing entity must be conceived in a scale-less fashion, and then presented as a goal for technologists across the planet, supported by, in the spirit of Feynman's way, a prize, for every realisation of this entity in every new, smaller, spatial scale. So, how does this "average Joe" telenanopresence robot will look like? First, let's consider the body of the robot. All we need to do to translate a human body into a scale-less robotic body is to first faithfully realise the human skeletal structure, using rods and joints etc., and then make this skeletal structure be able to withstand and exert the same, at scale, forces and torgues as our body naturally does, for every connection point of the skeleton. For example, let's say a human (for simplicity we assume humans are at the 1-meter scale) can exert at most 100 Newtons of force at the elbow when lifting something with the back kept upright. He uses this force to hold an object up, like a jug of water. The mass of this jug is related to its volume, so if our scale less robot needs to be able to hold up the same scale less jug, we need the force to scale as the volume scales with decreasing size. Thus the "milli-Joe", the telemilipresence robot roughly 1000 times smaller than a human, would have to be able to apply the equivalent force of 100 Newtons divided by the cube of the change in scale, so a billion times smaller. Assembling a set of structural and kinematic data about the human body and translating them for all target scales as specifications will be helpful for knowing when we have achieved satisfactory body telepresence in different scales. Another, considerations would be how accurately the movements of the human operator can be realised by the robotic body, and how quickly. The second area that we need to specify minimal requirements is the senses. The robot should be able to see, hear and feel, and send these data back to the human operator. Since the head of the robot will be able to move, two high definition cameras relaying stereoscopic vision back to the operator should be enough to build a virtual reality experience for him, and the same goes for hearing. Rudimentary feeling of objects should be achievable as well. If the robot can relay information about the relative angle of each joint of the robotic body, this 
information can be used to restrain the range of movement in a human operator's exoskeleton, giving haptic feedback. From the side of the human operator, the technology involved is the same as in virtual reality.

Motion tracking technology can be used to capture the movement of the operator and relay it to the robot, and an immersive experience of the environment where the robot is can be provided back to the operator, giving him a taste of how things work in a different spatial scale. In the spirit of the famous art pieces by legendary artist Joe Davis, "Audio Microscope" where he used a microscope to listen to protozo [2], and his ingenious Paramecia fishing contraption, where he rigged a fishing rod in mechanico-electronic feedback to a microscopic fishing hook which was thrown in a drop of pond water, extending human experience to the realms of the tiny will surely enhance our appreciation of the beauty of our natural world and especially the beauty of biology. At the same time, an entity of smaller and smaller size but with the same amount of brainpower might prove to be a fantastic innovator at the vast scales bellow. If one of our self-imposed purposes on this earth is to microfabricate, what better way to start it than to first make ourselves? To conclude, we propose the creation of a set of prizes, to incentivise the creation of telenanopresence machines. The prize would be given for the first robot build to the scale less specifications, that is smaller than a previously unattainable, new spatial scale. The division by 10 should be a good gap between each prize, so we can have a clear metric view of the categories of machines we have achieved building. This prize can be transformative for the technologies involved, in the same way that, for example, X-Prize has guided growth in space technology.

\section{References}

1. Feynman RP (1960) There is plenty of room at the bottom. Caltech Engineering and Science 23(5): 22-36.

2. Davis J. Online artist bio. Signed, Angelina Papachatzaki and Giannis Tamvakis. 